\title{
The protective effects of carboxyhemoglobin during the resuscitation from hemorrhagic shock in rats
}

\author{
Hongyu Liu, ${ }^{1, *}$, Songyan Yu ${ }^{2, *}$, Yaojun Peng ${ }^{3}$, Xin Chang ${ }^{4}$ and Xinguang $\mathbf{Y u}^{1}$ \\ ${ }^{1}$ Department of Neurosurgery, Chinese PLA General Hospital, Beijing 100853, China \\ ${ }^{2}$ Department of Endocrinology, Chinese PLA General Hospital, Beijing 100853, China \\ ${ }^{3}$ Key Laboratory of Cancer Center, Chinese PLA General Hospital, Beijing 100853, China \\ ${ }^{4}$ Department of Clinical Laboratory, Weihai Municipal Hospital, Weihai 264200, Shandong, China \\ *Co-first authors
}

Correspondence to: Xinguang Yu, email: xinguang_yu@263.net

Keywords: resuscitation from hemorrhagic shock, CO-red blood cells resuscitation, oxidative injury, inflammatory reaction

Received: November 02, $2016 \quad$ Accepted: March 21, $2017 \quad$ Published: June 28, 2017

Copyright: Liu et al. This is an open-access article distributed under the terms of the Creative Commons Attribution License 3.0 (CC BY 3.0 ), which permits unrestricted use, distribution, and reproduction in any medium, provided the original author and source are credited.

\section{ABSTRACT}

Aim: This study was aimed to explore the effects of carboxyhemoglobin on reperfusion injury in hemorrhagic shock, as well as its action time and related mechanisms.

Results: CO-RBC group showed milder oxidative injury than O2-RBC group. CO reperfusion did not show advantages in functions of kidney and lung during resuscitation. The level of Bax was decreased in CO-RBC group, especially in early CO-RBC group. Moreover, the autophay-related gene Beclin-1 was down-regulated in CO-RBC and early CO-RBC groups. The inflammation was severer in CO-RBC resuscitation group.

Materials and Methods: The hemorrhagic shock model rats were randomly divided into: the hemorrhagic shock group $(n=6)$; the 02-red blood cells $\left(\mathrm{O}_{2}-\mathrm{RBC}\right)$ group $(n=6)$, perfused with $O_{2}-R B C 1 \mathrm{~h}$ after ischemia; CO-RBC group $(n=12)$, perfused with CO-RBC $1 \mathrm{~h}$ after ischemia; and early CO-RBC group $(n=12)$, perfused with CO-RBC $30 \mathrm{~min}$ after ischemia. The reperfusion injuries were evaluated through antireactive oxygen species (ROS), inflammatory action, organ function, cell apoptosis and autophagy.

Conclusions: Carboxyhemoglobin not only relieves the oxidative injury and inhibites apoptosis and autophagy, but also aggravates inflammatory reactions during reperfusion. The action time of carboxyhemoglobin may be an influencing factor for reperfusion outcomes.

\section{INTRODUCTION}

Hemorrhagic shock is easily induced by bleeding from trauma, rupture of esophageal varices, peptic ulcer in clinic $[1,2]$. Rapid blood supplement is the primary rescue measure, which can recover the microcirculatory perfusion and oxygen supply in a short time [3]. However, there will be uncontrolled systemic inflammatory reactions over blood transfusion, namely reperfusion injuries, which is characterized by a large number of oxygen free radicals, leading to organ damages and dysfunction $[4,5]$. Therefore, it is important to avoid reperfusion injury in patients with hemorrhagic shock.

Reperfusion injury is a complex process with the involvement of oxidative stress, inflammatory response, cell apoptosis, autophagy, etc. However, the mechanisms underlying reperfusion injury remain unclear. Recently, accumulating studies are devoted to reduce reperfusion injury. The application of carbon monoxide (CO) is one of the hotspots. Evidence has shown that the oxygen supply recovery is not the earliest and the most critical demand, which could be delayed by carboxyhemoglobin $(\mathrm{COHb})$ retransfusion during resuscitation from shock [6]. CO shows cytoprotective effects in hemorrhagic shock/ resuscitation animal model via inhibiting inflammatory reactions [7]. In addition, heme oxygenase 1 (HO-1) is well known to play protective roles in inflammatory reactions and oxidative damage $[8,9]$. $\mathrm{CO}$, as a byproduct of HO-1 catalysis, can activate the soluble guanylate cyclase and transform GTP into cGMP at molecular 
level, playing important roles in relaxing smooth muscles and inhibiting platelet aggregation $[10,11]$. Moreover, $\mathrm{CO}$ can also relieve the inflammatory reactions and suppress apoptosis via activating p38/MAPK signaling pathway [12]. In addition, it is reported that CO-RBC can improve the amelioration of hepatic ischemia-reperfusion injury induced by hemorrhage and resuscitation via hepatic CYP protection [13]. However, no studies have comprehensively explored the role of $\mathrm{COHb}$ on reperfusion injury and its related mechanisms.

This study was attempted to explore the protective function of $\mathrm{CO}$ in reperfusion injury, as well as its effects on oxidative damage, inflammatory reactions, organ function, cell apoptosis and autophagy using the hemorrhagic shock/ resuscitation rat model. The resuscitation was implemented with infusion of autoblood mixed with $\mathrm{CO}$. The current study might explore the role of $\mathrm{CO}$ in resuscitation from hemorrhagic shock and its related mechanisms which might provide a new insight into the management of reperfusion in hemorrhagic shock patients.

\section{RESULTS}

\section{The decreased activities of RBC-SOD and lung- POD with $\mathrm{COHb}$ interference}

Oxidative injury is a major portion of the ischemia reperfusion injury, which was assessed by two antioxidases SOD and POD in the study. The SOD activity was higher in $\mathrm{O}_{2}-\mathrm{RBC}$ resuscitation group $(10341.16 \pm 4576.96)$ than those in hemorrhagic shock group (5999.33 \pm 6151.11$)$ and CO$\mathrm{RBC}$ resuscitation group (4555.44 \pm 3880.48$)$. In addition, the SOD activity in early $\mathrm{CO}-\mathrm{RBC}$ resuscitation group $(3359.28 \pm 2768.96)$ was close to that in CO-RBC group (Figure 1A). The results of POD activity in all groups were similar to those of SOD (Figure $1 \mathrm{~B}$ ). $\mathrm{COHb}$ significantly decreased the activities of SOD and POD in the hemorrhagic shock group compared with $\mathrm{O}_{2}-\mathrm{RBC}$ resuscitation group, and early or delayed CO-RBC resuscitation showed no difference on antioxidation (Figure 1).

\section{The increased expression of $T N F-\alpha, I L-1 \beta$, and $I L-6$ at mRNA level with $\mathrm{COHb}$ intervention}

The study measured the mRNA expression of several pro-inflammatory factor $N F-\alpha, I L-1 \beta$, and $I L-6$ in spleen to estimate the inflammatory reaction-caused tissue injury, which was also a portion of ischemia reperfusion injury. Generally speaking, the levels of proinflammatory factors in $\mathrm{O}_{2}-\mathrm{RBC}$ resuscitation group were lower than those in hemorrhagic shock group and $\mathrm{CO}$ $\mathrm{RBC}$ resuscitation group, and the later two groups had close expression levels of all factors except that $I L-1 \beta$ expression was higher in $\mathrm{CO}-\mathrm{RBC}$ blood group than hemorrhagic shock group $(P<0.05)$. Besides, the levels of pro-inflammatory factors were lower in early CO-RBC resuscitation group than delayed $\mathrm{CO}-\mathrm{RBC}$ resuscitation group. In short, $\mathrm{COHb}$ increased the mRNA levels of pro-inflammatory factors in hemorrhagic shock group compared with $\mathrm{O}_{2}-\mathrm{RBC}$ group, and expression of these factors was lower in early CO-RBC group than delayed CO-RBC group (Figure 2).

\section{The effects of $\mathrm{COHb}$ on functions of kidney and lung in resuscitation from hemorrhagic shock}

Blood urea nitrogen (BUN) concentration a main indicator that could reflect the renal glomerulus filtration and other renal functions. In the study, BUN concentration was used to assess the influence of ischemia reperfusion on kidney function and determine the effects of $\mathrm{COHb}$ during the process. The BUN concentration in $\mathrm{O}_{2}-\mathrm{RBC}$ resuscitation group $(6.60 \pm 1.86)$ was lower than that in hemorrhagic shock group $(7.19 \pm 1.71)$, and close to that in CO-RBC resuscitation group (6.63 \pm 1.10$)$. Meanwhile, early CO-RBC resuscitation group $(6.21 \pm 0.81)$ showed low BUN concentration compared with CO-RBC resuscitation group (Figure $3 \mathrm{~A}$ ). $\mathrm{COHb}$ showed no influence on BUN concentration during resuscitation from hemorrhagic shock, however, the BUN concentration was lower in early $\mathrm{CO}-\mathrm{RBC}$ resuscitation group rather than delayed $\mathrm{CO}-\mathrm{RBC}$ resuscitation group.

Pulmonary edema is the main feature of acute lung injury induced by ischemia reperfusion. In the study, the effects of $\mathrm{COHb}$ on resuscitation from hemorrhagic shock was evaluated by the wet-dry ratio, an index of pulmonary edema. The lung wet-dry ratio was lower in $\mathrm{O}_{2}-\mathrm{RBC}$ resuscitation group $(4.07 \pm 0.45)$ than hemorrhagic shock group ( $4.24 \pm 0.28)$, and close to CO-RBC resuscitation group (3.99 \pm 0.62$)$. In addition, similar lung wet-dry ratios were observed between early $\mathrm{CO}-\mathrm{RBC}$ resuscitation group $(4.02 \pm 0.57)$ and $\mathrm{CO}-\mathrm{RBC}$ resuscitation group (Figure 3B). COHb had no influence on pulmonary edema, and there was no difference in lung wet-dry ratio between early $\mathrm{CO}-\mathrm{RBC}$ resuscitation group and delayed $\mathrm{CO}-\mathrm{RBC}$ resuscitation group.

\section{Down-regulation of Bax mRNA and Beclin-1 mRNA in lung tissues with $\mathrm{COHb}$ intervention}

It is known that the majority of cells are apoptotic during the resuscitation from hemorrhagic shock. Thus, the present study determined the expression of Bax mRNA, a apoptosis-related gene, to investigate the effects of $\mathrm{COHb}$ on apoptosis in ischemia reperfusion injury. As shown in Figure 4A, the level of Bax mRNA was lower in $\mathrm{O}_{2}-\mathrm{RBC}$ resuscitation group $(0.56 \pm 0.28)$ than hemorrhagic shock group $(0.59 \pm 0.31)$, but higher than $\mathrm{CO}-\mathrm{RBC}$ resuscitation group $(0.50 \pm 0.20)$. In addition, the Bax mRNA was significantly elevated in early CO$\mathrm{RBC}$ resuscitation group compared with $\mathrm{CO}-\mathrm{RBC}$ resuscitation group. 
Autophagy represented a common stress response in cells. Beclin-1, an autophagy-related gene, was used to explore the effects of $\mathrm{COHb}$ on autophagy in ischemia reperfusion injury in the study. The expression of Beclin-1 mRNA in $\mathrm{O}_{2}-\mathrm{RBC}$ resuscitation group $(0.33 \pm 0.19)$ was close to that in hemorrhagic shock group $(0.33 \pm 0.13)$, while the data was higher than that in $\mathrm{CO}-\mathrm{RBC}$ resuscitation group $(0.26 \pm 0.13)$.
Moreover, the Beclin-1 mRNA level in early CO-RBC resuscitation group $(0.27 \pm 012)$ was close to that of CO-RBC resuscitation group (Figure 4B). The Beclin-1 mRNA expression was significantly down-regulated by $\mathrm{COHb}$ in hemorrhagic shock group compared with blood resuscitation group, which had no difference between early CO-RBC resuscitation and delayed CO-RBC resuscitation.
A

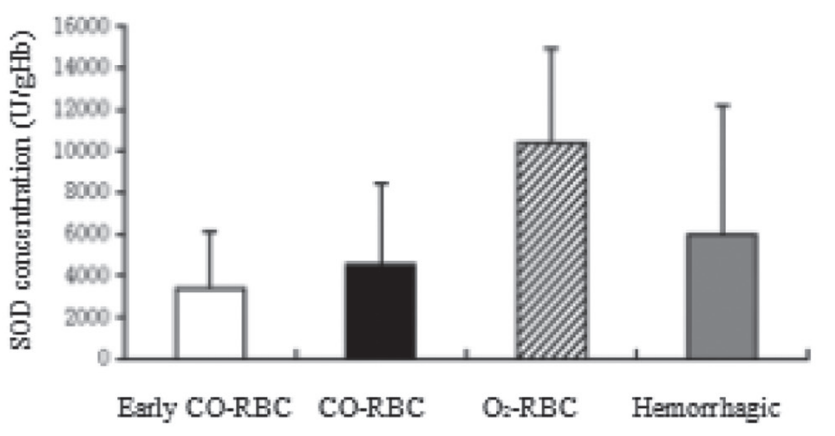

B

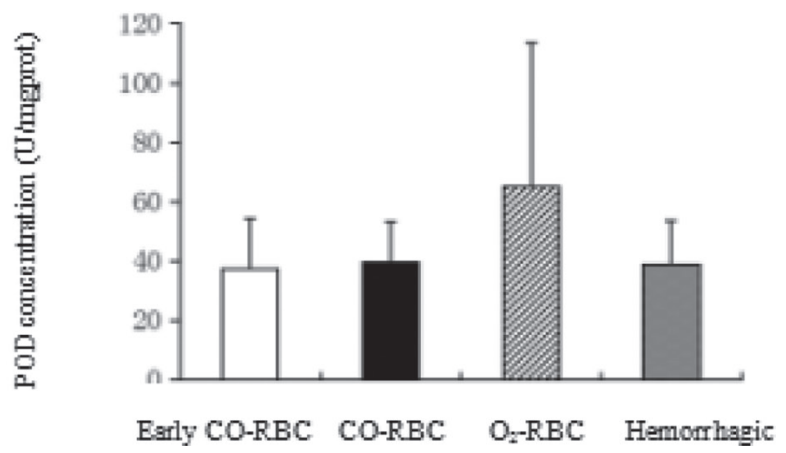

Figure 1: The effects of $\mathrm{COHb}$ on the antioxidative system during resuscitation from hemorrhagic shock. The abdominal aortic blood was collected from the rats to detect the SOD activity. And a part of the right lung was selected to determine the POD activity. (A) SOD activity in RBC. (B) POD activity in lung tissues. Data were shown as mean \pm SD.

A

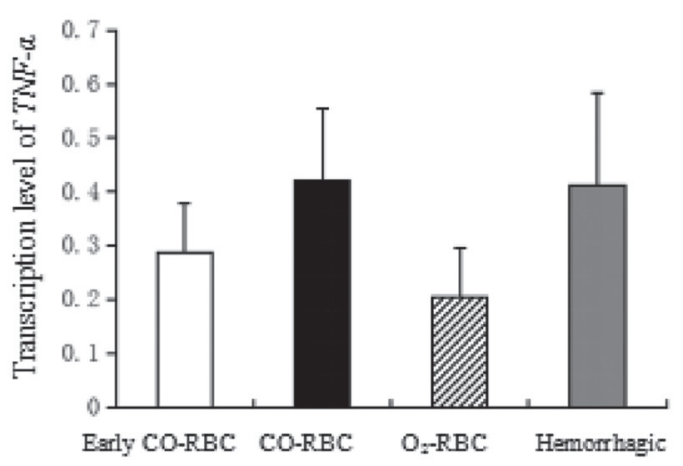

C

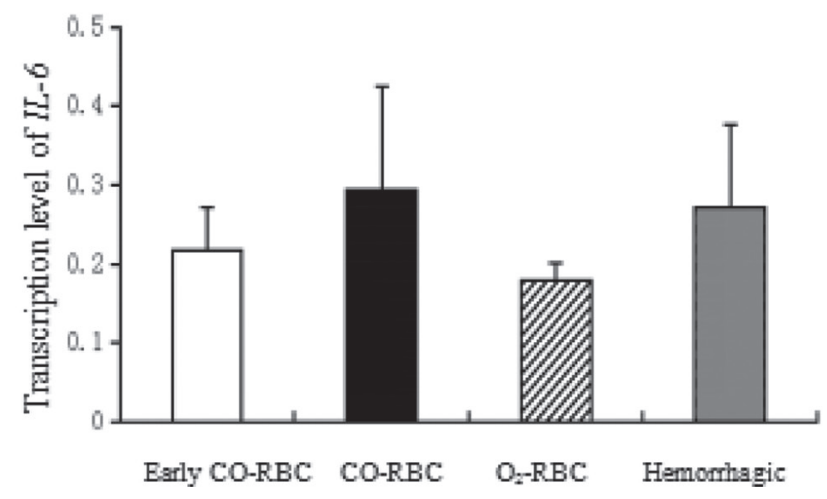

B

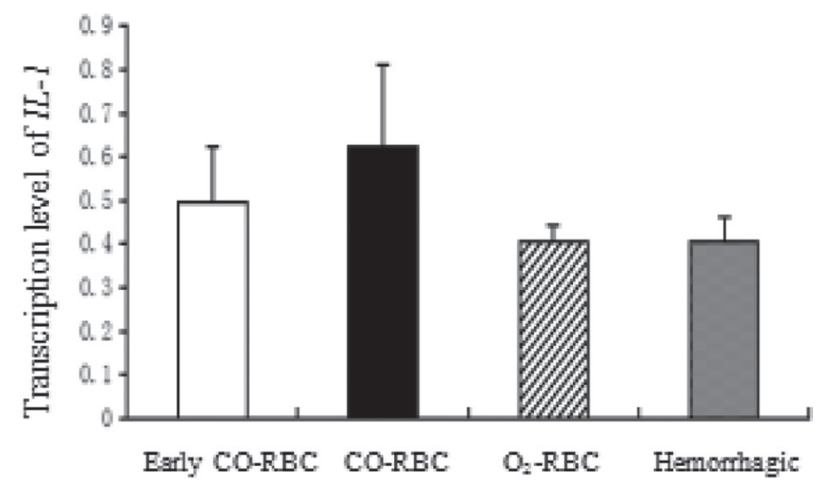

Figure 2: The effects of COHb on mRNA expression of $T N F-\alpha, I L-1 \beta$, and $\boldsymbol{I L}-\boldsymbol{6}$. All these genes were extracted from the spleen and determined using RT-PCR. Data were presented as mean $\pm \mathrm{SD}$. 


\section{DISCUSSION}

Oxidative damage is caused by a large number of oxygen free radicals which are generated with the recovery of oxygen supply after resuscitation from hemorrhagic shock, including lipid peroxidation of cell membrane, denaturation of proteins and nucleic acids [14]. Previous studies revealed that antioxidases had high affinity and reaction rate with oxygen free radicals [15]. In the current study, two antioxidases SOD and POD were selected as indicators to evaluate the oxidative damages reduced by resuscitation. The results showed that the activities of SOD and POD were both higher in $\mathrm{O}_{2}-\mathrm{RBC}$ resuscitation group. And the activity of SOD was lower in early CO-RBC and $\mathrm{CO}-\mathrm{RBC}$ resuscitation groups. All of the data suggested that $\mathrm{COHb}$ could suppress the generation of oxygen free radicals through delaying the recovery of oxygen supply, thus reducing the oxidative injury. However, the specific damage should be assessed by further detection of malonaldehyde (MDA) level in tissues, which was also one of the limitations in the study.

Inflammatory reaction is considered as a pivotal reason for reperfusion injury. As a main organ of lymphocytes and monocytes, spleen can promote the production and release of inflammatory cytokines in early acute inflammatory reactions, thus the transcription of pro-inflammatory cytokines in the spleen may be applied to evaluate systemic inflammatory reactions $[16,17]$. Previously researches indicated that $\mathrm{CO}$ could alleviate oxidative damage and inflammatory stress through activating p38 MAPK pathway $[18,19]$. In our study, the transcription levels of pro-inflammatory cytokines were higher in CO-RBC resuscitation group than $\mathrm{O}_{2}-\mathrm{RBC}$ resuscitation group, but similar to hemorrhagic shock group, which was different from the previous studies. It is well known that $\mathrm{CO}$ is a toxic gas can not be ignored, and the affinity between $\mathrm{CO}$ and hemoglobin is 240 times higher than that of oxygen, and it can inhibit the release of oxygen. Based on the data, we speculated that $\mathrm{CO}$ caused tissue hypoxia, hypoxia induced necrosis, and then enhanced inflammatory response. However, further investigations were required to address the related issues.

Ischemia reperfusion injury can induce multiple organ dysfunction syndrome (MODS). The study investigated the role of $\mathrm{COHb}$ application in organ dysfunction during resuscitation from hemorrhagic shock
A

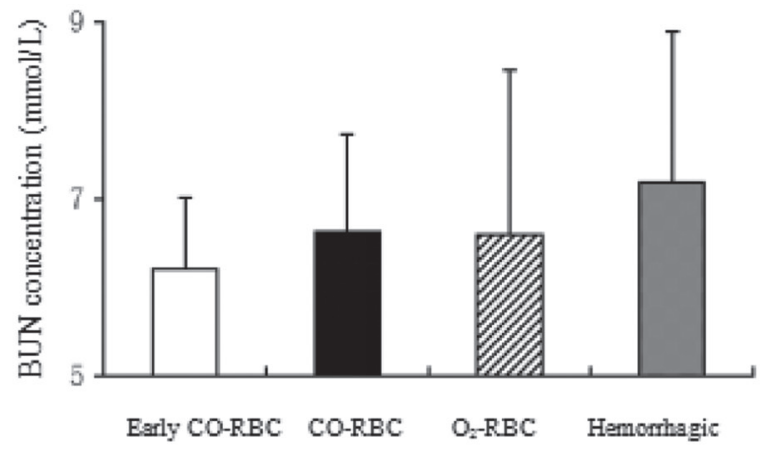

B

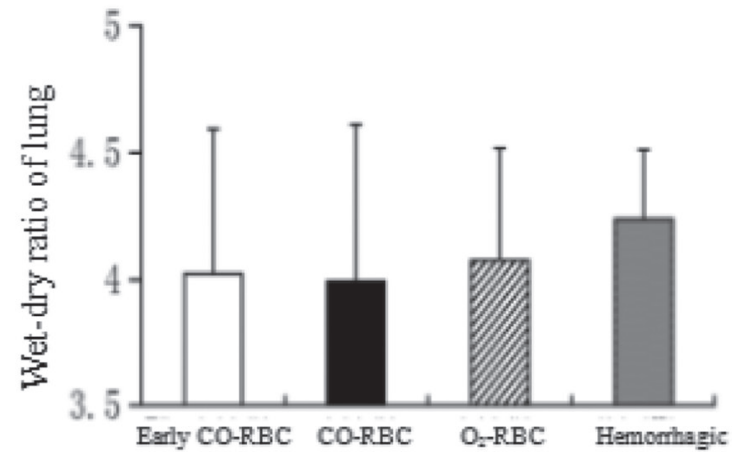

Figure 3: The influence of $\mathrm{COHb}$ on the functions and structures of kidney and lung. The BUN concentration was determined with the abdominal aortic blood and the wet-dry ratio of lung was calculated with the left lung. (A) BUN concentration. (B) Wet-dry ratio of lung. All data were shown as mean \pm SD.

A

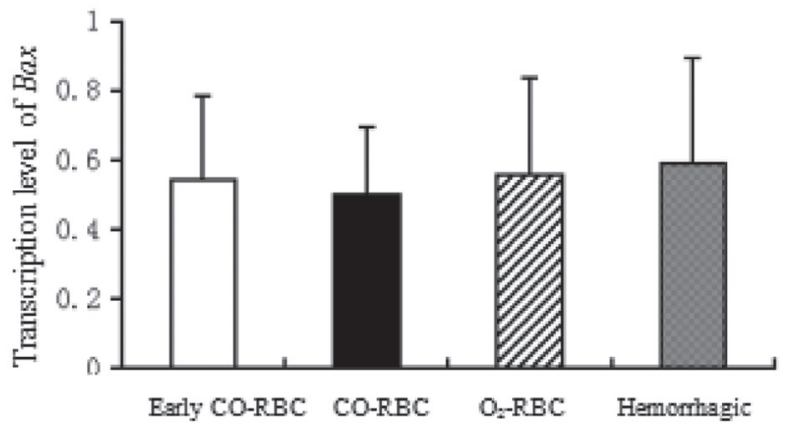

B

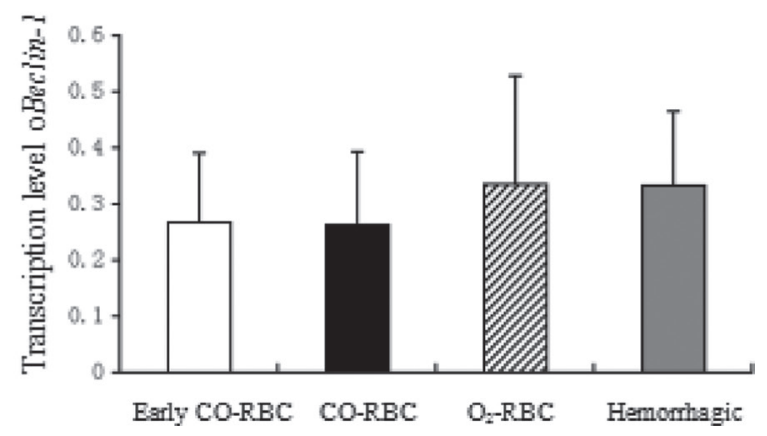

Figure 4: The effects of COHb on expression of Bax mRNA and Beclin-1 mRNA. Bax and Beclin-1 were extracted from lung tissues and determined by RT-PCR using quantity one software. All data were presented as mean \pm SD. 
via evaluating the structure and function of kidney and lung. The results showed that $\mathrm{CO}-\mathrm{RBC}$ resuscitation group and $\mathrm{O}_{2}-\mathrm{RBC}$ resuscitation group had similar BUN concentration and lung wet-dry ratio, and both showed better structure and function of kidney and lung than hemorrhagic shock group. The renal compensation ability was strong and the BUN concentration would be increased massively only when the filtration rate of kidney glomerulus decreased to below $50 \%$ of normal values. Therefore, the ischemia reperfusion injury of kidney in our study might remain within its compensation ability (renal ischemia reperfusion). The present study did not find the protective effect of $\mathrm{CO}$ on lung, which might be related with the different ischemia reperfusion modes of between our study and previous studies.

Moreover, our study found that the transcription level of Bax in CO-RBC resuscitation group was lower than $\mathrm{O}_{2}-\mathrm{RBC}$ resuscitation group, which was accordant with the cell apoptosis detection conducted on these three groups after resuscitation by Pedro et al. [6]. And the results indicated that $\mathrm{COHb}$ could inhibit cell apoptosis, but the mechanisms were poorly known.

Beclin-1 is a marker protein for autophagy in mammalians and participates in the formation of autophagosome membrane. Hence, the transcription level of Beclin-1 is positively correlated with the induction level of autophagy. Studies have suggested that autophagy played a protective role in the ischemia reperfusion injury of the heart through cleaning the damaged mitochondria [20]. However, in the current study we found that the expression of autophagy-related gene was lower in CO-RBC group. Autophagy has dual characters. Some studies explained that autophagy could protect the cells during ischemia, but went against resuscitation during reperfusion, owing to the accumulation of mitochondria with structural and functional disorders in the damaged cells during ischemia, which might make autophagic responses more aggressive and lead to cell death during resuscitation $[21,22]$. Thus, further investigations were still needed to explore the role of autophagy in resuscitation from hemorrhagic shock and the relationship between the expression of related-genes and $\mathrm{CO}$ reperfusion.

Whole blood resuscitation can recover the oxygen supply, which corrects the ischemia and hypoxia, but initiates oxidative injury. That is to say bodies presented comprehensive results of hypoxia injury and oxidative damage. The human $\mathrm{COHb}$ intervention can not only delay the recovery of oxygen supply and reduce oxidative damages but also recover blood supply and eventually accomplish reoxygenation. The appropriate action time of $\mathrm{COHb}$ is critical for the reperfusion outcomes. In this study, we found that the levels of cell apoptosis gene Bax were significantly lower in early $\mathrm{CO}-\mathrm{RBC}$ group than that in the CO-RBC group, revealing that early application of
$\mathrm{CO}$ reperfusion exhibited enhanced protective ability in cell apoptosis. Furthermore, we also found that the levels of inflammatory cytokines presented decreased trend in early CO-RBC group. The early application of CO might also suppress inflammatory reactions. Consequently, in order to improve the clinical outcomes of reperfusion among patients with hemorrhagic shock, further investigations were still needed to optimize the strategy for application of CO-RBC.

In conclusion, $\mathrm{COHb}$ plays important protective roles in oxidative injury and cell apoptosis through delaying the recovery of oxygen supply in ischemic tissues. However, $\mathrm{COHb}$ may aggravate inflammatory reactions. The outcomes of reperfusion may be influenced by the action time of $\mathrm{COHb}$.

\section{MATERIALS AND METHODS}

\section{Animals and reagents}

Male SD rates (average body weight $293.61 \mathrm{~g}$ ) were purchased from the Experimental Animal Center of the Academy of Military Medical Science. All animal studies were approved by the Institutional Care and Use Committee of the hospital.

The $10 \%$ chloral hydrate, $70 \mathrm{U} / \mathrm{ml}$ heparin sodium, recombinant human serum albumin (rHSA), total superoxide dismutase (T-SOD) Kit, peroxidase (POD) Kit, blood urea nitrogen (BUN) Kit and total protein Kit was purchased from Nanjing Jiancheng Bioengineering Institute. The Quantscript RT Kit and 2XTaq PCR Master Mix Kit were obtained from Tiangen Biotech (Beijing) Co., Ltd.

\section{Animal treatments and preparation of hemorrhagic shock model rats}

A total of 36 adult male SD rats were randomly divided into 4 groups: hemorrhagic shock group (Hemorrhagic, $n=6$ ), $\mathrm{O}_{2}$-red blood cells resuscitation group $\left(\mathrm{O}_{2}-\mathrm{RBC}, n=6\right)$, $\mathrm{CO}-\mathrm{RBC}$ resuscitation group $(\mathrm{CO}-\mathrm{RBC}, n=12)$ and early $\mathrm{CO}-\mathrm{RBC}$ resuscitation group (early CO-RBC, $n=12$ ). All animals were weighted and then anesthetized by $10 \%$ chloral hydrate (peritoneal injection, $0.3 \mathrm{ml} / 100 \mathrm{~g}$ ). After that, the rats were fixed lying on the operation table. The right external jugular vein was selected from the median neck incision and injected with heparin sodium (70U/100 g), hemostasis by compression. The neck muscles were operated with blunt dissection to separate the left common carotid artery near the trachea. Blood withdrawal from the artery was complemented in $5 \mathrm{~min}$ using a $10 \mathrm{ml}$ syringe and about $30 \%$ of the total blood volume (calculated as $6 \%$ of body weight) was collected. Then arterial occlusion was conducted. 
Table 1: The primer sequences of related genes

\begin{tabular}{ccc}
\hline Genes & Upstream primer (Sense) & Downstream primer (Antisense) \\
\hline TNF- $\alpha$ & 5'-AGGCTCCTCTCCGCCATCAAGA-3' & 5'-TGGGCTCATACCAGGGCTT-3' \\
IL-1 $\beta$ & 5'-GATGGCTGCACTATTCCTAATGC-3' & 5'-AGACTGCCCATTCTCGACAAG-3' \\
IL-6 & 5'-TAGTCCTTCCTACCCCAACTTCC-3' & 5'-TTGGTCCTTAGCCACTCCTTC-3' $^{\prime}$ \\
Bax & 5'-GGCGAATTGGAGATGAACTG-3' & 5'-GTCACTGTCTGCCATGTGGG-3' $^{\prime}$ \\
Beclin-1 & 5'-GGAGATGTTGGAGCAAATGAA-3' & 5'-GTCGCATTGAAGACATTGGTT-3' $^{\prime}$ \\
GAPDH & 5'-CCACCACCATCTTCCAGGAG-3' & 5'-CCTGCTTCACCACCTTCTTG-3' \\
\hline
\end{tabular}

\section{Preparation of $\mathrm{COHb}$}

The blood samples from donor rats were withdrawn into heparinized syringes $(0.15 \mathrm{~mL}$ of $10,000 \mathrm{IU} / \mathrm{mL}$ heparin to $10 \mathrm{~mL}$ of blood) and centrifuged; it was then washed twice by resuspension in 5\% rHSA and centrifugation (3000 g, $10 \mathrm{~min})$. The formic acid and concentrated sulfuric acid (3:2) were mixed and then heated to prepare $\mathrm{CO}$ gas. When the $\mathrm{CO}$ concentration reached $1 \%(10000 \mathrm{ppm})$, mixed with balanced air $(21 \%$ oxygen), and the concentration of $\mathrm{CO}$ was maintained at $250 \mathrm{ppm}$. The $\mathrm{Hb}$ of $\mathrm{O}_{2}-\mathrm{RBC}$ was adjusted to $8.6 \mathrm{~g} / \mathrm{dL}$. The $\mathrm{CO}-\mathrm{RBC}$ was prepared using gentle $\mathrm{CO}$ bubbling for approximately $5 \mathrm{~min}$. The levels of $\mathrm{COHb}$ in the blood were detected by OSM3 Hemoximeter (Radiometer Copenhagen, Copenhagen, Denmark).

\section{Resuscitative reperfusion}

Half the draw-out blood (normal whole blood or CO-containing whole blood) was reperfused into the right external jugular vein. Rats in Hemorrhagic group were without any treatment; $\mathrm{O}_{2}-\mathrm{RBC}$ group rats were infused the normal whole blood into the right external jugular vein when the rats had ischemia $1 \mathrm{~h}$. CO-RBC group were infused the $\mathrm{CO}$-containing whole blood into the right external jugular vein when the rats had ischemia $1 \mathrm{~h}$. Early CO-RBC group were infused the CO-containing whole blood when the rats had ischemia $30 \mathrm{~min}$.

\section{Sample extraction of testing indexes}

The spleen was resected from the median abdominal incision $1 \mathrm{~h}$ after resuscitation (or $2 \mathrm{~h}$ after ischemia in the hemorrhagic shock group) and stored in liquid nitrogen for determining the mRNA expression of $T N F-\alpha, I L-1 \beta$, and $I L-6$. An aliquot of $2 \mathrm{ml}$ blood was withdrawn from the abdominal aorta to detect the BUN concentration and RBC-SOD activity. Both left and right lungs were excised by opening the thoracic cavity. Part of the right lung was stored in liquid nitrogen to examine the transcription of Bax, Bcl-2, Beclin-1, and the rest was used to detect the POD activity of lung tissues. The left lung was used to measure the wet-dry ratio of lung.

\section{Detection of antioxidase activity}

Red blood cell extract was prepared by centrifugation of portal vein blood with $2 \mathrm{ml}$, then the erythrocyte SOD activity was detected by the nitrite formation method according to the instruction of total superoxide dismutase (T-SOD) Kit (Nanjing Jiancheng Bioengineering Institute).

After all the experiments, $1 \mathrm{~g}$ lung tissue of rats were collected and treated with cold $5 \% \mathrm{CaCl}_{2}$ solution, then put it in an ice water bath, the quality of the final concentration of $2 \%$. Afterwards, centrifugation for $20 \mathrm{~min}$ in $4^{\circ} \mathrm{C}, 12000 \mathrm{rpm}$, collecting supernatant, then using guaiacol colorimetric method to assay the activity of POD according to the instruction of peroxidase (POD) Kit (Nanjing Jiancheng Bioengineering Institute).

\section{Detection of blood urea nitrogen (BUN) concentration}

After preparation of plasma samples, the BUN concentration was determined using the Fearon method.

\section{Wet-dry ratio of lung}

The left lung was wiped by absorbent paper and weighed as wet weight. Then the lung was dried at $70^{\circ} \mathrm{C}$ for $12 \mathrm{~h}$ and weighted as dry weight. The wet-dry ratio could serve as an indicator for pulmonary edema.

\section{Quantitative real-time polymerase chain reaction (RT-PCR)}

Total RNA was isolated from the rats' spleens and lungs as previously described. The two-step PCR was performed. PCR conditions for $T N F-\alpha, I L-1 \beta$, and $I L-6$ were as follows: pre-denaturation at $95^{\circ} \mathrm{C}$ for 30 s, denaturation at $95^{\circ} \mathrm{C}$ for $5 \mathrm{~s}$, annealing at $60^{\circ} \mathrm{C}$, and extension at $60^{\circ} \mathrm{C}$ for $34 \mathrm{~s}, 30$ cycles. And PCR for Bax, Beclin-1 was taken under the following conditions: predenaturation at $94^{\circ} \mathrm{C}$ for $4 \mathrm{~min}$, denaturation at $94^{\circ} \mathrm{C}$ for $30 \mathrm{~s}$, annealing at $55^{\circ} \mathrm{C}($ Bax $)$ or $51^{\circ} \mathrm{C}($ Beclin-1), and extension for 30 s, 30 cycles. GAPDH served as internal control, and the sequences of primers were summarized in Table 1. 


\section{Statistical analysis}

All data were presented as mean \pm SD and analyzed using the Sigmaplot 12.5 software (Systat Software Inc., CA, USA). $P<0.05$ was considered to be significant.

\section{ACKNOWLEDGMENTS}

None.

\section{CONFLICTS OF INTEREST}

None.

\section{GRANT SUPPORT}

None.

\section{REFERENCES}

1. Cherkas D. Traumatic hemorrhagic shock: advances in fuid management. Emerg Med Pract. 2011; 13:1-19; quiz 19-20.

2. Abassi ZA, Okun-Gurevich M, Abu Salah N, Awad H, Mandel Y, Campino G, Mahajna A, Feuerstein GZ, Fitzpatrick M, Hoffman A, Winaver J. Potential early predictors for outcomes of experimental hemorrhagic shock induced by uncontrolled internal bleeding in rats. PloS one. 2013; 8:e80862.

3. Ortiz D, Barros M, Yan S, Cabrales P. Resuscitation from hemorrhagic shock using polymerized hemoglobin compared to blood. Am J Emerg Med. 2014; 32:248-255.

4. Kalogeris T, Baines CP, Krenz M, Korthuis RJ. Cell biology of ischemia/reperfusion injury. Int Rev Cell Mol Biol. 2012; 298:229-317.

5. Gillani S, Cao J, Suzuki T, Hak DJ. The effect of ischemia reperfusion injury on skeletal muscle. Injury. 2012; 43:670-675.

6. Cabrales P, Tsai AG, Intaglietta M. Hemorrhagic shock resuscitation with carbon monoxide saturated blood. Resuscitation. 2007; 72:306-318.

7. Kanagawa F, Takahashi T, Inoue K, Shimizu H, Omori E, Morimatsu H, Maeda S, Katayama H, Nakao A, Morita K. Protective effect of carbon monoxide inhalation on lung injury after hemorrhagic shock/resuscitation in rats. J Trauma. 2010; 69:185-194.

8. Yang Y, Wang J, Li Y, Fan C, Jiang S, Zhao L, Di S, Xin Z, Wang B, Wu G, Li X, Li Z, Gao X, et al. HO-1 Signaling Activation by Pterostilbene Treatment Attenuates Mitochondrial Oxidative Damage Induced by Cerebral Ischemia Reperfusion Injury. Mol Neurobiol. 2016; 53:2339-2353.

9. Yeligar SM, Machida K, Kalra VK. Ethanol-induced HO-1 and NQO1 are differentially regulated by HIF-1alpha and $\mathrm{Nrf} 2$ to attenuate infammatory cytokine expression. J Biol Chem. 2010; 285:35359-35373.
10. Schallner N, Romao CC, Biermann J, Lagreze WA, Otterbein LE, Buerkle H, Loop T, Goebel U. Carbon monoxide abrogates ischemic insult to neuronal cells via the soluble guanylate cyclase-cGMP pathway. PloS one. 2013; 8:e60672.

11. Chlopicki S, Lomnicka M, Fedorowicz A, Grochal E, Kramkowski K, Mogielnicki A, Buczko W, Motterlini R. Inhibition of platelet aggregation by carbon monoxidereleasing molecules (CO-RMs): comparison with NO donors. Naunyn Schmiedebergs Arch Pharmacol. 2012; 385:641-650.

12. Nakao A, Choi AM, Murase N. Protective effect of carbon monoxide in transplantation. J Cell Mol Med. 2006; 10:650-671.

13. Ogaki S, Taguchi K, Watanabe H, Ishima Y, Otagiri M, Maruyama T. Carbon monoxide-bound red blood cell resuscitation ameliorates hepatic injury induced by massive hemorrhage and red blood cell resuscitation via hepatic cytochrome $\mathrm{P} 450$ protection in hemorrhagic shock rats. J Pharm Sci. 2014; 103:2199-2206.

14. Weight SC, Bell PR, Nicholson ML. Renal ischaemiareperfusion injury. Br J Surg. 1996; 83:162-170.

15. Esposito E, Cuzzocrea S. Role of nitroso radicals as drug targets in circulatory shock. Br J Pharmacol. 2009; 157:494-508.

16. Bachman SL, Hanly EJ, Saad D, Nwanko JI, Lamb J, Herring AE, Marohn MR, De-Maio A, Talamini MA. The role of the spleen in laparoscopy-associated infammatory response. Surg Endosc. 2005; 19:1035-1044.

17. Bao Y, Kim E, Bhosle S, Mehta H, Cho S. A role for spleen monocytes in post-ischemic brain infammation and injury. J Neuroinflammation. 2010; 7:92.

18. Brugger J, Schick MA, Brock RW, Baumann A, Muellenbach RM, Roewer N, Wunder C. Carbon monoxide has antioxidative properties in the liver involving p38 MAP kinase pathway in a murine model of systemic infammation. Microcirculation. 2010; 17:504-513.

19. Li Y, Gao C, Shi Y, Tang Y, Liu L, Xiong T, Du M, Xing M, Yao P. Carbon monoxide alleviates ethanolinduced oxidative damage and infammatory stress through activating p38 MAPK pathway. Toxicol Appl Pharmacol. 2013; 273:53-58.

20. Hamze A, Rasolofonjatovo E, Provot O, Mousset C, Veau D, Rodrigo J, Bignon J, Liu JM, Wdzieczak-Bakala J, Thoret S, Dubois J, Brion JD, Alami M. B-ring-modifed isocombretastatin A-4 analogues endowed with interesting anticancer activities. ChemMedChem. 2011; 6:2179-2191.

21. Yan L, Sadoshima J, Vatner DE, Vatner SF. Autophagy: a novel protective mechanism in chronic ischemia. Cell Cycle. 2006; 5:1175-1177.

22. Sciarretta S, Hariharan N, Monden Y, Zablocki D, Sadoshima J. Is autophagy in response to ischemia and reperfusion protective or detrimental for the heart? Pediatr Cardiol. 2011; 32:275-281. 